The possibility of setting $p_{k}=0, k>0$, for large values of $t$ will depend on whether $\phi(t)$ admits an asymptotic expansion along the positive real axis. The region of definition of $\phi(t)$ as well as the possibility of its expansion by the transformation (11) may be inferred from general theorems in the theory of divergent series*. With due regard to the distribution of the singularities of $f(p),(6)$ and (11) may be used when $f(p)$ is expanded in a two-way Laurent series.

RUTGERS UNIVERSITY

\title{
SYMMETRIC, SELF-DUAL, RATIONAL PLANE CURVES OF ODD ORDER $\dagger$
}

BY D. C. DUNCAN

The writer has established the existence $\$$ of the real, nondegenerate, completely symmetric, self-dual, elliptic curves of order $4 k+2$, and has indicated an extremely simple plan of sketching the loci approximately. In pursuing the program there indicated, we now establish the existence of completely symmetric rational self-dual curves of order $2 k+1$, and indicate the corresponding easy mode of sketching them approximately. These loci also are invariant under $4 k+2$ collineations and $4 k+2$ correlations of which $2 k+1$ are polarities by real rectangular hyperbolas and one a polarity by an imaginary circle. Moreover, the $(2 k+1)^{2}$ foci have an interesting distribution and admit rather easy determination. In conclusion, the equations in Cartesian coordinates of the curves of the lowest three orders, 5, 7, and 9, are listed, together with a sketch of the 9-ic depicting all singular elements, real foci, and real autopolarizing conics. In a subsequent paper the elliptic and rational self-dual completely symmetric curves of orders $4 k, 2 k+1$, and $4 k, 4 k+2$, respectively, are to be exhibited, therewith terminating the present discussion.

* See, for example, Borel et Bouligand, Leçons sur les Séries Divergents, Gauthier-Villars, 1928, Chapter 4.

$\dagger$ Presented to the Society under a slightly different title, December 2, 1933.

$\ddagger$ This Bulletin, vol. 39 (1933), pp. 809-813 
Plücker's formulas show that the self-dual, rational curve of order $2 k+1$ has $2 k-1$ cusps. In the present treatment, as in the elliptic case, we let these $2 k-1$ cusps be distributed at equal intervals about the unit circle. Moreover, let the cuspidal tangents be concurrent at the center of the circle, namely at the point $(0,0,1)$ of a homogeneous rectangular coordinate system. Let these cuspidal tangents be axes of symmetry, and assume the locus to be invariant under rotations about $(0,0,1)$ through angles of $2 \pi m /(2 k-1),(m=1,2, \cdots, 2 k-2)$. As an immediate consequence of this last assumption, we see that the $(k-1)(2 k-1)$ nodes must lie by $(k-1)$ 's on each of these $2 k-1$ cuspidal tangents.

The most general equation of order $2 k+1$ in homogeneous rectangular coordinates is of the form

$$
\begin{aligned}
A f_{2 k+1}(x, y) & +B f_{2 k}(x, y) z+C f_{2 k-1}(x, y) z^{2} \\
& +\cdots+K f_{1}(x, y) z^{2 k}+L z^{2 k+1}=0 .
\end{aligned}
$$

The various $f_{i}(x, y)$ are homogeneous polynomial functions of degree $i$ in $x, y$, which must individually be absolutely invariant under the rotations about the origin through angles of $2 \pi m /(2 k-1)$. Since $f_{i}(x, y)=0$ represents $i$ straight lines through the origin it is clearly impossible for a set of $i$ straight lines to be invariant under rotations of $2 \pi /(2 k-1)$ if $i<(2 k-1)$, unless they are the isotropic lines $\left(x^{2}+y^{2}\right)^{h}=0$. Hence

$$
\begin{aligned}
f_{2 q}(x, y) & \equiv\left(x^{2}+y^{2}\right)^{q}, \quad(q=1,2, \cdots, k) ; \\
f_{2 q-1}(x, y) & \equiv 0, \quad(q=1,2, \cdots, k-1) .
\end{aligned}
$$

Accordingly, if $y=0$ is a cuspidal tangent, and consequently an axis of symmetry, the equation of the locus in polar coordinates must be

$$
\begin{gathered}
A \rho^{2 k+1} \cos (2 k-1) \theta+B \rho^{2 k}+C \rho^{2 k-1} \cos (2 k-1) \theta \\
+D \rho^{2 k-2}+E \rho^{2 k-4}+\cdots+H \rho^{2}+L=0 .
\end{gathered}
$$

When $\theta=0$, the roots of

$$
A \rho^{2 k+1}+B \rho^{2 k}+C \rho^{2 k-1}+D \rho^{2 k-2}+\cdots+H \rho^{2}+L=0
$$

must include a triple root for $\rho=1$, the cusp, and double roots for $a_{1}, a_{2}, a_{3}, \cdots, a_{k-1}$, the nodes. We next observe that for 
just one essential set of real values of $A, B, \cdots, H, L$, the equation can have such roots. Assume

$$
\begin{aligned}
A \rho^{2 k+1}+B \rho^{2 k}+ & \cdots+H \rho^{2}+L \\
& \equiv A(\rho-1)^{3}\left(\rho-a_{1}\right)^{2}\left(\rho-a_{2}\right)^{2} \cdots\left(\rho-a_{k-1}\right)^{2} .
\end{aligned}
$$

We here require the $a$ 's to be distinct, none vanishing or equal to unity, in order that the singularities may all be simple. Since the coefficients of $\rho, \rho^{3}, \rho^{5}, \cdots, \rho^{2 k-3}$ are zero, one has necessarily $\sum_{r}\left(1,1,1, a_{1}, a_{1}, a_{2}, \cdots, a_{k-1}\right)=0$, where $\sum_{r}(1,1,1$, $\left.a_{1}, \cdots, a_{k-1}\right)$ represents the sum of the products of the roots $r$ at a time, $(r=4,6,8, \cdots, 2 k)$, there being $k-1$ such equations. Now let these various $a_{i}$ be replaced by their reciprocals and the resultant equations cleared of fractions; this will not impair the solutions since by hypothesis $a_{i} \neq 0$. One then obtains the set of equations

$$
\sum_{r^{\prime}}\left(1,1,1, a_{1}^{\prime}, a_{1}^{\prime}, a_{2}^{\prime}, a_{2}^{\prime}, \cdots, a_{k-1}^{\prime}, a_{k-1}^{\prime}\right)=0,
$$

$\left(r^{\prime}=1,3,5, \cdots, 2 k-3\right),\left(a^{\prime}=1 / a\right)$. These equations may now be replaced by the simpler ones*

$$
\sum_{1}\left(1,1,1, a_{1}^{\prime s}, a_{1}^{\prime s}, a_{2}^{\prime s}, a_{2}^{\prime s}, \cdots, a_{k-1}^{\prime s}, a_{k-1}^{\prime s}\right)=0
$$

$(s=1,3,5, \cdots, 2 k-3)$, that is,

$$
\begin{aligned}
3+2 a_{1}^{\prime}+2 a_{2}^{\prime}+\cdots+2 a_{k-1}^{\prime} & =0 \\
3+2 a_{1}^{\prime 3}+2 a_{2}^{\prime 3}+\cdots+2 a_{k-1}^{\prime 3} & =0 \\
3+2 a_{1}^{\prime 5}+2 a_{2}^{\prime 5}+\cdots+2 a_{k-1}^{\prime 5} & =0
\end{aligned}
$$

or

$$
3+2 a_{1}^{\prime 2 k-3}+2 a_{2}^{\prime 2 k-3}+\cdots+2 a_{k-1}^{2 k-3}=0,
$$

$$
\begin{array}{r}
a_{1}^{\prime}+a_{2}^{\prime}+a_{3}^{\prime}+\cdots+a_{k-1}^{\prime}=-3 / 2, \\
a_{1}^{\prime 3}+a_{2}^{\prime 3}+a_{3}^{\prime 3}+\cdots+a_{k-1}^{\prime 3}=-3 / 2, \\
a_{1}^{\prime 5}+a_{2}^{\prime 5}+a_{3}^{\prime 5}+\cdots+a_{k-1}^{\prime 5}=-3 / 2, \\
\cdot \cdot \cdot \cdot \cdot \cdot \cdot \cdot \cdot \cdot \cdot \cdot \cdot \cdot \cdot \cdot \cdot \cdot \cdot \cdot \cdot \\
a_{1}^{2 k-3}+a_{2}^{\prime 2 k-3}+a_{3}^{\prime 2 k-3}+\cdots+a_{k-1}^{\prime 2 k-3}=-3 / 2 \cdot \dagger
\end{array}
$$

* Cajori, Theory of Equations, p. 85, application of Newton's formulas.

$\dagger$ Thus, for $k=4, a_{1}^{\prime}=-.679523 \cdots, a_{2}^{\prime}=-1.062793 \cdots, a_{3}{ }^{\prime}=.242317 \cdots$. 
Regard these $k-1$ equations as representing $k-1$ hypersurfaces in $(k-1)$-space, and consider the distances from the origin, $(0,0,0, \cdots, 0)$, to the intersections of the line $a_{1}^{\prime}=a_{2}^{\prime}=a_{3}^{\prime}=\ldots=a_{k-1}^{\prime}$ with them; these distances are proportional to $[-3 / 2((k-1))]^{1 / p},(p=1,3,5, \cdots, 2 k-3)$. We observe that these distances are of increasing order of magnitude. Now consider the distances from the origin to the intersections of these hypersurfaces with an axis of coordinates, say, $a_{2}=0, a_{3}=0, \cdots, a_{k-1}=0$. These distances are, respectively, $[-3 / 2]^{1 / p},(p=1,3,5, \cdots, 2 k-3)$. Note that these distances are of decreasing order of magnitude. Hence from considerations of continuity one observes the existence of a common solution of these equations, no value of $a_{i}$ being zero. On account of the perfect symmetry of the $a$ 's there are $(k-1)$ ! solutions arising by permuting the $a$ 's in all possible ways. We next prove the uniqueness of this fundamental solution. Assume two distinct fundamental solutions; then there would be two different sets of values of $A, B, C, \cdots, L$, in the equation of the locus, that is, letting $A$ and $A^{\prime}=1$,

and

$$
\begin{aligned}
\Phi \equiv & \rho^{2 k+1} \cos (2 k-1) \theta+B \rho^{2 k}+C \rho^{2 k-1} \cos (2 k-1) \theta \\
& +D \rho^{2 k-2}+\cdots+H \rho^{2}+L=0
\end{aligned}
$$

$$
\begin{aligned}
\Phi^{\prime} \equiv & \rho^{2 k+1} \cos (2 k-1) \theta+B^{\prime} \rho^{2 k}+C^{\prime} \rho^{2 k-1} \cos (2 k-1) \theta \\
& +D^{\prime} \rho^{2 k-2}+\cdots+H^{\prime} \rho^{2}+L^{\prime}=0 .
\end{aligned}
$$

If we set $\theta=0$, the form $\Phi-\Phi^{\prime}$ must not contain any odd powers . of $\rho$ except $2 k+1$ and $2 k-1$. Factoring, we obtain $\Phi-\Phi^{\prime}$ in the form

$$
\begin{aligned}
(\rho-1)^{3}\left[\left(\rho^{2 k-2}+B \rho^{2 k-4}+C \rho^{2 k-6}+\cdots+H \rho^{2}+L\right)\right. \\
\left.-\left(\rho^{2 k-2}+B^{\prime} \rho^{2 k-4}+C^{\prime} \rho^{2 k-6}+\cdots+H^{\prime} \rho^{2}+L^{\prime}\right)\right] .
\end{aligned}
$$

The condition that the specified odd powers of $\rho$ vanish yields $B=B^{\prime}, C=C^{\prime}, \cdots, L=L^{\prime}$. Hence it follows that there exists only one essentially distinct set of values of $A, B, C, \cdots, L$. Accordingly, the locus $\Phi=0$ is unique. Hence if the points of inflexion and the cuspidal tangents, and also the cusps and the inflexional tangents, are pole-and-polar with respect to any conic section, this conic section will autopolarize the entire locus $\Phi=0$. Let us observe that the points at infinity on the lines 
$\theta=(4 m+2 k-1) \pi /(4 k-2),(m=0,1,2, \cdots, 2 k-2)$, are points of inflexion. In particular, consider the inflexion at infinity on the $y$-axis, that is, for $m=0$. The inflexional tangent at this point, $(0,1,0)$, is $(2 k-1) x \pm B z=0,(+\operatorname{sign}$ before $B$ if $k$ is even, - sign if $k$ is odd). We observe, then, that the polarity $u: v: w=(2 k-1) x:(2 k-1) y: \pm B$ interchanges the inflexional and cuspidal elements at $(0,1,0)$ and $(1,0,1)$. By symmetry, all inflexional and cuspidal elements are pole-and-polar with respect to the same conic, namely, $(2 k-1)\left[x^{2}+y^{2}\right] \pm B z^{2}=0$. But since $y$ occurs only with even powers in the equation $\Phi=0$, the locus $\Phi=0$ is also invariant under polarization with respect to the rectangular hyperbola $(2 k-1)\left(x^{2}-y^{2}\right) \pm B z^{2}=0$, and hence, by symmetry, also with respect to the rectangular hyperbolas symmetrically placed with reference to the other cuspidal tangents, namely, $2 k-1$ hyperbolas in all. Combining one of these polarities with a reflexion about each of the $2 k-1$ cuspidal tangents (axes of symmetry) yields $2 k-2$ correlations, which are not polarities, and the polarity with respect to the imaginary circle. The products of any correlation by all the correlations are the $2(2 k-1)$ collineations. They may be represented explicitly as follows.

\section{Collineations}

$$
\begin{aligned}
x & =x \cos 2 \pi m /(2 k-1) \mp y \sin 2 \pi m /(2 k-1), \\
y & =x \sin 2 \pi m /(2 k-1) \pm y \cos 2 \pi m /(2 k-1), \\
z & =z, \quad(m=0,1,2, \cdots, 2 k-2) .
\end{aligned}
$$

\section{Correlations (Polarities)}

$$
\begin{aligned}
u & =(2 k-1) x, \quad v=(2 k-1) y, \quad w= \pm B z, \\
u & =(2 k-1)[x \cos (2 \pi m /(2 k-1))-y \sin (2 \pi m /(2 k-1))], \\
v & =(2 k-1)[-x \sin (2 \pi m /(2 k-1))-y \cos (2 \pi m /(2 k-1))], \\
w & = \pm B z,
\end{aligned}
$$

\section{Correlations (Not Polarities)}

$$
u=(2 k-1)[x \cos (2 \pi m /(2 k-1))+y \sin (2 \pi m /(2 k-1))],
$$$$
v=(2 k-1)[-x \sin (2 \pi m /(2 k-1))+y \cos (2 \pi m /(2 k-1))] \text {, }
$$$$
w= \pm B z, \quad(m=1,2,3, \cdots, 2 k-2) \text {. }
$$

[Throughout, + attaches to $B$ if $k$ is even, - if $k$ is odd.] 
The form of the locus is apparent from the following considerations. Since there are as many inflexions (all at infinity) as there are cusps, each arm of each cusp can cut an arm of another cusp no more than once. Each of the $2 k-1$ arms extending from cusp to cusp can intersect $2 k-2$ others only in $2 k-4$ points in addition to the two cusps; that is, in $(2 k-1)(k-2)$ nodes, which, by symmetry, must be distributed equally on the $2 k-1$ cuspidal tangents; hence $k-2$ nodes are on each cuspidal tangent, whereas there should be $k-1$. Therefore one node on each cuspidal tangent must be an acnode, and must lie within the unit circle; otherwise the circle passing through them would cut the locus in $8 k-4$ points, whereas there can be at most $2(2 k+1)$ intersections of a $(2 k+1)$-ic with a circle. For the same reason no acnodes may lie outside the unit circle, hence all nodes outside the unit circle are crunodes. This maximum number $k-2$ is possible only if each arm joining two cusps cuts both arms of all the other cusps; that is, the arms of adjacent cusps unite at an inflexion at infinity. Accordingly we may make the following statement.

The completely symmetric self-dual rational curve of order $2 k+1$, possessing, accordingly, $2 k-1$ cusps and $(2 k-1)(k-1)$ nodes, of which $2 k-1$ are acnodes, may be sketched very approximately by drawing the $2 k-1$ secant lines through consecutive pairs of $2 k-1$ points equally distributed on the circumference of the unit circle, omitting the chords within the circle. The $2 k-1$ corners may then be tapered to resemble cusps. The $2 k-1$ acnodes may be placed within the circle midway between consecutive cusps.

Since these loci are of order $2 k+1$ and have $(2 k-1)$-fold rotational symmetry, they all pass through the circular points at infinity. Moreover, the $(2 k+1)^{2}$ foci are distributed as follows. There are one real and $2 k$ distinct imaginary foci on each cuspidal tangent, namely, $\begin{aligned} x: y: z= & \operatorname{ir}\left[e^{2 m \pi i /(2 k+1)}+e^{2 m^{\prime} \pi i /(2 k+1)}\right]: r\left[e^{2 m \pi i /(2 k+1)}\right. \\ & \left.-e^{2 m^{\prime} \pi i /(2 k+1)}\right]: 2 i .\end{aligned}$

There are also two real and two imaginary foci coincident at the origin, $(0,0,1)$. The other imaginary foci occur in coincident pairs at the points

$$
x: y: z=r e^{2 m \pi i /(2 k+1)}: \pm i r e^{2 m \pi i /(2 k+1)}: 2,
$$

$\left(m, m^{\prime}=0,1,2, \cdots, 2 k\right)$. The foci are obtained by assuming 
the line through $(1: i: 0)$ and $(r: 0: 1)$ to be tangent to the locus, and then requiring the pole of this tangent with respect to a polarizing conic to lie on the locus; that is, one requires the coordinates $( \pm B i:-[ \pm B]:-i r[2 k-1])$ to satisfy the equation $\Phi=0$. The actual calculation is somewhat tedious although it is confined to terms of degrees $2 k+1$ and $2 k$. The equations of the curves arising for $k=1,2$, and 3 are listed with a brief discussion and sketch of that of degree 9, arising for $k=3$ :

$$
\begin{gathered}
n=5 \quad(k=1) \\
\left(9 x^{2}+9 y^{2}-5\right)\left(x^{3}-3 x y^{2}\right)-15\left(x^{2}+y^{2}\right)\left(x^{2}+y^{2}-1\right)-4=0 . \\
n=7 \quad(k=2) \\
\left(25 x^{2}+25 y^{2}-105\right)\left(x^{5}-10 x^{3} y^{2}+5 x y^{4}\right) \\
-105\left(x^{2}+y^{2}\right)^{3}-385\left(x^{2}+y^{2}\right)^{2}+420\left(x^{2}+y^{2}\right)=144 . \\
n=9 \quad(k=3) \quad[\text { homogeneous rectangular coordinates] } \\
49\left(x^{9}-20 x^{7} y^{2}+14 x^{5} y^{4}+28 x^{3} y^{6}-7 x y^{8}\right)-315\left(x^{2}+y^{2}\right)^{4} z \\
-45\left(x^{7}-21 x^{5} y^{2}+35 x^{3} y^{4}-7 x y^{6}\right) z^{2}+2415\left(x^{2}+y^{2}\right)^{3} z^{3} \\
-5544\left(x^{2}+y^{2}\right)^{2} z^{5}+5040\left(x^{2}+y^{2}\right) z^{7}-1600 z^{9}=0 .
\end{gathered}
$$

On the cuspidal tangent $y=0$ occur a cusp at (1:0:1), an acnode at $(-.9404917 \ldots: 0: 1)$, and crunodes at $(-1.47162 \cdots: 0: 1)$ and $(4.12682 \cdots: 0: 1)$. The equations of the tangents at these nodes are as follows:

$$
\begin{aligned}
& \pm 3.740 y=x-4.128 z \\
& \pm 0.106 i y=x+0.941 z \\
& \pm 1.257 y=x+1.419 z
\end{aligned}
$$

the coefficients are correct only to the third decimals as indicated. A point of inflexion occurs at $(0: 1: 0)$, the inflexional tangent [asymptote] at which is $49 x+45 z=0$. The two proper bitangents and one isolated bitangent which are perpendicular to the cuspidal tangent $y=0$ are, respectively,

$$
\begin{aligned}
(72.10938 \cdots) x-45 z & =0, \\
(202.21418 \cdots) x-5 z & =0, \\
(46.104933 \cdots) x-5 z & =0 .
\end{aligned}
$$

The coefficients again are correct tu we indicated places of decimals. The six other singular elemerits of each of these kinds are readily found by rotating thest through angles $2 \pi m / 7$, 
$(m=1,2,3, \cdots, 6)$. Although the point and line singularities are all distinct, certain of the foci coincide. One observes that there are one real and six distinct imaginary foci on each cuspidal tangent, namely at

$$
x: y: z=\operatorname{ir}\left[e^{2 \pi k^{\prime} i / 7}+e^{2 \pi k^{\prime} i / 7}\right]: r\left[e^{2 \pi k i / 7}-e^{2 \pi k^{\prime} i / 7}\right]:+2 i,
$$

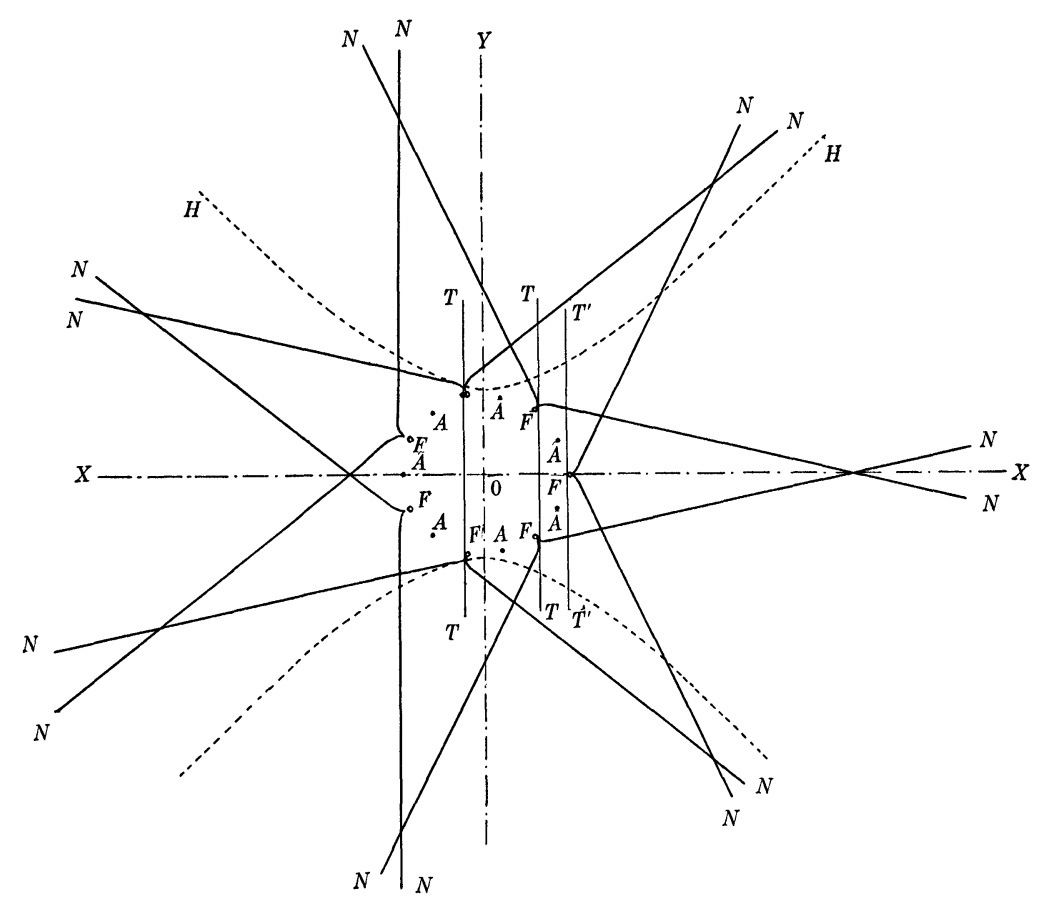

$N$ : Autopolar locus of order 9. $\cdot A$ : Acnode. $F$ : Real focus.

$H$ : One of 7 autopolarizing rectangular hyperbolas; the others are symmetrically placed.

$T T$ : Proper bitangent. $T^{\prime} T^{\prime}$ : Isolated bitangent.

besides two real and two imaginary coincident foci at the origin, $(0: 0: 1)$. The other imagivary foci occur in coincident pairs at the points,

$$
x: y: z=r e^{2 \pi k i / 7}: \pm i r e^{2 \pi \pi \cdot k i / 7}:+2, \quad\left(k, k^{\prime}=0,1,2, \cdots, 6\right),
$$

where $r=(45 / 49)(9 / 5)^{1 / 7}$. 
The 14 collineations and 14 correlations under which the curve is invariant may be written explicitly as follows:

\section{Collineations}

$$
\begin{aligned}
& x: y: z=x \cos (2 \pi k / 7) \theta+y \sin (2 \pi k / 7) \theta: x \sin (2 \pi k / 7) \theta \\
& +y \cos (2 \pi k / 7) \theta: z \text {. } \\
& \text { Correlations (Polarities) } \\
& u=49 x \quad u=49[x \cos (2 \pi k / 7) \theta-y \sin (2 \pi k / 7) \theta], \\
& v=49 y, \quad v=49[-x \sin (2 \pi k / 7) \theta-y \cos (2 \pi k / 7) \theta] \text {, } \\
& w=45 z, \quad w=45 z, \quad(k=0,1,2, \cdots, 6) \text {. } \\
& \text { Correlations (Not Polarities) } \\
& u=49[x \cos (2 \pi k / 7) \theta+y \sin (2 \pi k / 7) \theta] \text {, } \\
& v=49[-x \sin (2 \pi k / 7) \theta+y \cos (2 \pi k / 7) \theta] \text {, } \\
& w=45 z, \\
& (k=1,2, \cdots, 6) \text {. }
\end{aligned}
$$

The form of the curve is very approximately realized by drawing secant lines through consecutive points of the seven points equally distributed about the unit circle. The figure depicts all the singular elements and real autopolarizing conics.

The University of California 